\title{
Surface properties and stray fields of rf-sputtered $\mathrm{Co}-\mathrm{Cr}$ films
}

\author{
W.J.M.A. Geerts, J.C. Lodder and Th. J.A. Popma \\ Univ. of Twente, P.O. Box 217, 7500 AE Enschede, Netherlands
}

\begin{abstract}
The influence of surface properties on the recording performance of thin films is at best illustrated in the perpendicular recording mode. The roughness and the oxidated/segregated top layer will redistribute the magnetic charges at the surface. The stray field which is so important in the rcading process will deteriorate. Attention will be focussed on the relation between the stray field and magnetization (J.J. Miyata et al., IRE Trans. Elect. Comp. (1959) 159) as a function of the above mentioned properties. Using experimental data the reduction of the maximum readable information density is calculated.
\end{abstract}

The data used in this paper are obtained from rf sputtered $\mathrm{Co}-\mathrm{Cr}$ films having a perpendicular anisotropy [2].

The chemical composition of these films was determined by XPS and Auger depth profiling. The oxide segregation scales of our $\mathrm{Co}-\mathrm{Cr}$ films show a triplex character. At the surface only $\mathrm{CoO}$ could be observed while the inner oxide scale mainly consisted of $\mathrm{Cr}_{2} \mathrm{O}_{3}$. The difference of the metal's diffusion coefficients in the oxidized scale and the difference of the affinity for oxygen of both metals are responsible for the measured composition profiles [3].

The roughness of the films was determined by SEM and AFM experiments. SEM photographs showed a dome-like surface structure. The column diameter $(=$ $D$ ) appeared to depend on the film thickness $(=d)$, and was found to increase with the thickness $(40<D<$ $180 \mathrm{~nm}$ for $80<d<1200 \mathrm{~nm})$. SEM cross-section fractions showed a roughness height $(=h)$ of about $D / 3$. More accurate numerical values were obtained by performing AFM studies. Three-dimensional surface profiles were measured by scanning in the constant force mode. The roughness period, as determined from these pictures, coincides with $D_{\text {SEM }}$. The roughness height was determined from the width of the surface depth distribution function (=SDDF) [4] which was calculated from the measured surface profiles. Numerical values for $h$ varied between 5 and $50 \mathrm{~nm}$ for samples with a film thickness of 80 to $1200 \mathrm{~nm}$. The SDDFs were quadratic functions which is in agreement with theoretical calculations of a regular dome-like surface profile.
The measured composition profiles were transformed to Magnetic Charge Density Distribution (MCDD) functions by assuming:

1) Oxygen is preferentially bounded to $\mathrm{Cr}$ to form the non-magnetic $\mathrm{Cr}_{2} \mathrm{O}_{3}$.

2) The oxygen remaining after the reaction with $\mathrm{Cr}$ will reduce the magnetic moment of the Co $\left(M_{\mathrm{s}}\right.$ linear with the at $\%$ oxygen and zero for 33 at $\%$ oxygen as shown in ref. [5]).

3) The non-oxidized $\mathrm{Cr}$ will lower the magnetic moment of the $\mathrm{Co}\left(M_{\mathrm{s}}\right.$ lineair with the at $\% \mathrm{Cr}$ atoms and zero for 25 at $\% \mathrm{Cr}$ as shown in ref. [6]).

The calculated magnetization profilcs show the existence of a buried top layer with a saturation magnetization which is even higher than the bulk value [3]. The MCDD-function, the derivative of the $M_{\mathrm{s}}$ profile $\left(\rho_{\mathrm{m}}\right.$ $=-\boldsymbol{\nabla} \cdot \boldsymbol{M})$, can be approximated by two block functions with opposite signs (see fig. 1b).

The stray field integrated along the track width, and not the local stray field, is the important parameter for recording. The redistribution of the magnetization by the surface roughness can thus be considered to be proportional to the SDDF.

Because of the quadratic character of the SDDF the MCDD of a regular dome-like surface is a linear function of the distance from the point of observation to a virtual surface (see fig. 1c). Consequently the inhomogeneous composition at the surface and the roughness will lead to three-dimensional magnetic charge packets at the surface (fig. 2).

The MCDD functions of fig. 1 are composed of constant and linearly increasing contributions. By using
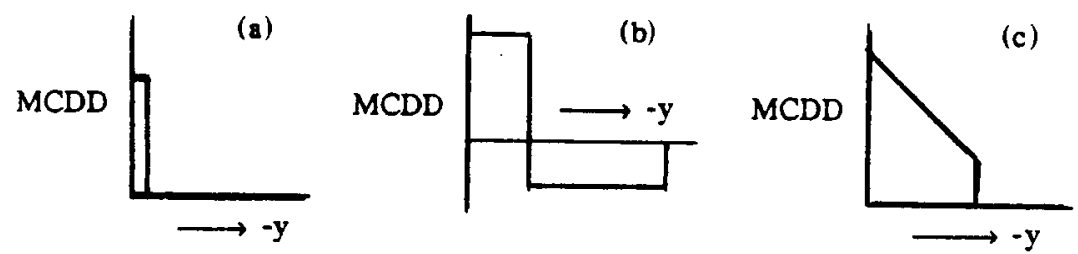

Fig. 1. Magnetic charge as a function of the distance from the point of observation to the surface. The film's surface is positioned at $-y=0$ while the substrate can be found at $-y=\infty$. In the case of (a) a smooth non-oxidized film, (b) an oxidized film, (c) a rough film. 
Film surface

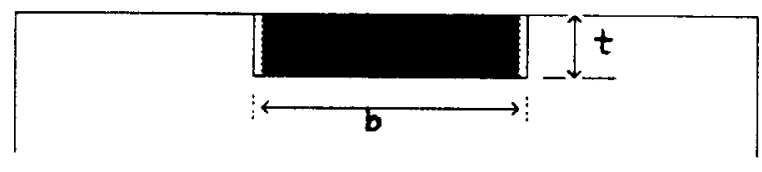

\section{= magnetic charge}

Fig. 2. Magnetic charge packet at the film's surface of a single bit in a demagnetized medium.

standard integration techniques we calculated the stray field from both types of contributions. The film surface is considered to be parallel to the $x-z$ plane. The track width along the $z$-direction is taken as infinite. The expressions were derived for the stray field in the $y$-direction $\left(H_{\text {con }}(x, y)\right.$ and $\left.H_{\text {lin }}(x, y)\right)$ above a magnetic charge packet of width $b$ and thickness $t$ (see fig. 2).

1) $\mathrm{MCDD}=\rho_{\text {con }}$ for $0<y<t$ and $\mathrm{MCDD}=0$ for all other $y$ :

$$
\begin{aligned}
H_{\text {con }}(x, y)= & \frac{\rho_{\text {con }}}{4 \pi}(A(y)+A(y+t)+B(x-b / 2) \\
& -B(x+b / 2)) .
\end{aligned}
$$

2) $\operatorname{MCDD}=\rho_{\text {lin }} y$ for $0<y<t$ and $\mathrm{MCDD}=0$ for all other $y$ :

$$
\begin{aligned}
H_{\text {lin }}(x, y)= & -2 \frac{\rho_{\text {lin }}}{4 \pi}\left(C\left(x+\frac{b}{2}\right)-C\left(x-\frac{b}{2}\right)-\frac{b}{t}\right) \\
& +\left.H_{\text {con }}(x, y)\right|_{\rho_{\text {con }}-\rho_{\operatorname{lin}},},
\end{aligned}
$$

with the functions $A, B$ and $C$ defined as:

$$
\begin{aligned}
& A(a)=2 a\left(\operatorname{atan} \frac{x+b / 2}{a}-\operatorname{atan} \frac{x-b / 2}{a}\right), \\
& B(a)=x \ln \frac{y^{2}+a^{2}}{(y+t)^{2}+a^{2}}, \\
& C(a)=\left(a^{2}+y^{2}\right) \operatorname{atan} \frac{a}{y}\left(a^{2}+(y+t)^{2}\right) \operatorname{atan} \underset{y+t}{a} .
\end{aligned}
$$

These equations were used in order to calculate the stray field above rough or oxidized $\mathrm{Co}-\mathrm{Cr}$ films. The stray field was analysed in terms of its amplitude, i.e. the maximum stray field along the $y$-direction $(=\mathbf{F} \Lambda)$, and its pulse width ( $=\mathrm{PW}$, the $20 \%$ pulse width [1]). Both parameters depend on the bit size and the distance above the film $(=y)$. The results given below are obtained for contact recording $(y=0)$. The calculations are made for two bit sizes, i.e. $b=100 \mathrm{~nm}$ which is nowadays almost achievable in a laboratory set-up

\begin{tabular}{|c|c|c|c|c|c|}
\hline$d$ & $d_{\mathrm{SEM}}$ & $d_{\mathrm{AFM}}$ & $h_{\text {AFM }}$ & $\begin{array}{l}\text { BDL } \\
(b=10)\end{array}$ & $\begin{array}{l}\text { BDL } \\
(b=100)\end{array}$ \\
\hline 80 & 60 & 50 & 5 & & \\
\hline 1200 & 180 & 180 & 50 & $87 \%$ & $53 \%$ \\
\hline $40 / 1200$ & \multicolumn{3}{|c|}{$\begin{array}{l}t_{\mathrm{l}}=2, t_{2}=6 \\
M_{\mathrm{S}}(\text { surf }) / M_{\mathrm{s}} \text { (bulk) } \\
=2.5\end{array}$} & $30 \%$ & $<5 \%$ \\
\hline
\end{tabular}
[7], and $b=10 \mathrm{~nm}$ a future bit size. The contributions of the charges at the film substrate interface are not included in our calculations (= no thickness loss). The
Table 1

Topology and chemical composition at the surface (all sizes in $\mathrm{nm})$

influcnce of an oxidized scale on FA and PW was small for the $100 \mathrm{~nm}$ bit size. For the $10 \mathrm{~nm}$ bit size the FA decreased by $20 \%$ while the PW increased by about $25 \%$. The influence of the measured roughnes on the stray field was much larger. For the roughest surface in the case of the $10 \mathrm{~nm}$ bit sizc the FA was about $20 \%$ of that above a completely smooth surface. The PW was even 5 times larger than the bit width.

A recording system can be considered as a communication channel (with the past). The influence of the above calculated stray-field changes upon the maximum readable information density can be estimated by using Shannon's theory [8] ( = relation between maximum bit density and PW, FA and $\triangle F A \quad(=$ mathematical variation of $F A)$ ). The parameter $\triangle F A$ can be calculated by assuming a maximum bit density for a smooth and unoxidized film. Using this value and the parameters FA and PW, which are influenced by the surface properties, the maximum information density for the $\mathrm{Co}-\mathrm{Cr}$ films presented here was estimated. The last row of table 1 presents the bit density loss in comparison with a non-oxidized smooth film. If the bit size reaches the order of magnitude of the surface inhomogencitics, the maximum readable information density is largely decreased. It can thus be concluded that, in the near future, storage technology will most likely depend on controlling the surface properties of the media, not only for lubrication and durability reasons [4], but also from the point of view of information density.

The authors would like to thank P. van Heeren from Thermo Instrument Systcms B.V. (Brcda, The Netherlands) for assisting with the AFM experiments.

\section{References}

[1] J.J. Miyata et al., IRE Trans. Elect. Comp. (1959) 159.

[2] W.J.M.A. Geerts et al., IEEE Trans. Magn. MAG-26 (1990) 36.

[3] T. Masuda et al., J. Magn. Magn. Mater. 95 (1991) 123.

[4] Bharat Bhushan, Tribology and Mechanics of Magnetic Storage Devices (Springer, New York, 1990) p. 73.

[5] N. Niratsuka et al., Trans. IECE Jpn. J72-C (1989) 237.

[6] Parcas, Ann. Rev. Phys. 8 (1937) 146.

[7] S.B. Luitjens et al., IEEE Trans. Magn. MAG-24 (1988) 2338.

[8] C.E. Shannon, Theory on Communication (Univ. of Illinois Press, Urbana, 1963). 